J. Indones. Math. Soc.

Vol. 23, No. 2 (2017), pp. 67-75.

\title{
FULL IDENTIFICATION OF IDEMPOTENTS IN BINARY ABELIAN GROUP RINGS
}

\author{
Kai Lin Ong${ }^{1}$, Minn Huey Ang ${ }^{2}$ \\ ${ }^{1,2}$ Pusat Pengajian Sains Matematik, \\ Universiti Sains Malaysia, Penang, Malaysia \\ 1..am.kailin@hotmail.com \\ ${ }^{2}$ mathamh@usm.my
}

\begin{abstract}
Every code in the latest study of group ring codes is a submodule that has a generator. Study reveals that each of these binary group ring codes can have multiple generators that have diverse algebraic properties. However, idempotent generators get the most attention as codes with an idempotent generator are easier to determine its minimal distance. We have fully identify all idempotents in every binary cyclic group ring algebraically using basis idempotents. However, the concept of basis idempotent constrained the flexibilities of extending our work into the study of identification of idempotents in non-cyclic groups. In this paper, we extend the concept of basis idempotent into idempotent that has a generator, called a generated idempotent. We show that every idempotent in an abelian group ring is either a generated idempotent or a finite sum of generated idempotents. Lastly, we show a way to identify all idempotents in every binary abelian group ring algebraically by fully obtain the support of each generated idempotent.
\end{abstract}

Key words and Phrases: idempotent, generated idempotent, group ring code, binary abelian group ring.

2000 Mathematics Subject Classification: 11T71, 94B99.

Received: Received: 20 Aug 2016, revised: 12 July 2017, accepted: 17 July 2017. 


\begin{abstract}
Abstrak. Setiap kode dalam kajian kode gelanggang grup didefinisikan sebagai suatu submodul yang mempunyai pembangun. Telah diketahui bahwa setiap kode gelanggang grup biner dapat memiliki banyak pembangun yang kaya akan sifatsifat aljabar. Dari sekian banyak pembangun ini, pembangun idempoten adalah jenis pembangun yang paling menarik, karena kode dengan pembangun idempoten mudah ditentukan jarak minimumnya. Telah diidentifikasi semua idempoten dari setiap gelanggang grup biner yang siklik secara aljabar dengan menggunakan basis idempoten. Akan tetapi, konsep basis idempoten membatasi fleksibilitas untuk memperluas kajian ke arah identifikasi idempoten untuk kasus tak-siklik. Di dalam paper ini, diperluas konsep basis idempoten menjadi idempoten yang memiliki pembangun, yang disebut dengan idempoten yang dibangun(generated idempotent). Kemudian, ditunjukkan bahwa idempoten pada suatu gelanggang grup yang komutatif adalah berbentuk generated idempotent atau jumlah hingga dari generated idempotent. Lebih jauh, ditunjukkan juga suatu cara untuk mengidentifikasi semua idempoten di setiap gelanggang grup yang komutatif secara aljabar dengan cara menentukan semua support dari setiap generated idempotent.
\end{abstract}

Kata kunci: Idempoten,generated idempotent, kode gelanggang grup, gelanggang grup biner komutatif.

\title{
1. INTRODUCTION
}

In this paper, $G$ is referred as a finite abelian group. Let $F_{q}$ be a finite field of $q$ elements. A group ring of $G$ over $F_{q}$ is the set $F_{q} G=\left\{\sum_{g \in G} a_{g} g \mid a_{g} \in F_{q}\right\}$. The support of a group ring element $u=\sum_{g \in G} a_{g} g \in F_{2} G$ is denoted as $\operatorname{supp}(u)=$ $\left\{g \in G \mid a_{g} \neq 0\right\}$. Furthermore, if $u \in F_{q} G$ satisfying $u^{2}=u$, then $u$ is called an idempotent in $F_{q} G$. Every group ring $F_{q} G$ has at least two idempotents, namely the trivial idempotents, which are 0 and 1.

Since 1967, S.D. Berman introduced the classical notion of $F_{q} G$ codes, which are basically ideals of $F_{q} G$ [1]. For decades, having idempotents as generators of $F_{q} G$ code has been widely proven to be an essence in finding the codes' minimum distance $[3,9,10,11]$. In 2006, Ted Hurley and Paul Hurley introduced a modern approach to study $F_{q} G$ codes, which are submodules of the $F_{q} G$ [4]. Toward this direction, the resultant $F_{q} G$ codes are categorized into two main families, namely the zero-divisor codes and unit-derived codes, with respect to whether their generator is a zero divisor or unit. Some researches show that the zero-divisor codes are good sources of self-dual codes $[2,4,6,8]$. In fact, the non-trivial idempotents are also zero-divisors. This inspires us to study the potential of having idempotent generators for zero-divisor codes in determining their properties. However, in an arbitrary group ring, it is not an easy task to identify those idempotents from a large pool of group ring elements.

In [7], we have fully identify all idempotents in every $F_{2} C_{n}$ algebraically, where $C_{n}$ is a cyclic group, by using our concept of basis idempotents. We show 
that every $F_{2} C_{n}$ will have a unique idempotent, $e_{L}$ with largest support size. We introduce a way to partition the support of $e_{L}$ into the supports of some idempotents which we defined as basis idempotents. The set of linear combination of the basis idempotents over $F_{2}$ is then shown to be exactly the set of all idempotents in $F_{2} C_{n}$. However; the definition of our basis idempotents constrained the flexibilities of extending our work into the study of identification of all idempotents in $F_{2} G$, where $\mathrm{G}$ is non-cyclic.

In this paper, we extend our concept of basis idempotents in $F_{2} C_{n}$ into our concept of generated idempotents in $F_{2} G$. In Section 2, we introduce the generated idempotents as well as their important properties. We show that every idempotent in $F_{2} G$ is either a generated idempotent or a finite sum of some generated idempotents and there exists a unique largest finite sum of generated idempotents in $F_{2} G$. In Section 3, we show the largest odd order subgroup of $G$ forms the support of the largest finite sum of generated idempotents in $F_{2} G$. We also prove that the support of all non-zero generated idempotents in $F_{2} G$ can be obtained by partitioning the support of the largest finite sum of generated idempotents and thus we identify all the idempotents in $F_{2} G$.

\section{Generated Idempotents}

Recall that $G$ is referred as a finite abelian group. Let $I(G)$ denote the set of all idempotents in $F_{2} G$. The closure property on the addition of the set is affirmed by the following proposition.

Proposition 2.1. The set $I(G)$ is closed under addition.

Proof. Let $e_{1}, e_{2} \in I(G)$, then $\left(e_{1}+e_{2}\right)^{2}=e_{1}^{2}+2 e_{1} e_{2}+e_{2}^{2}=e_{1}+e_{2}$.

Next we introduce a special type of permutation that help in differentiating elements in $I(G)$ from $F_{2} G$.

Definition 2.2. Let $a \in F_{2} G$. Define $\varphi_{a}: \operatorname{supp}(a) \rightarrow G$ such that $\varphi_{a}(g)=g^{2}$. If $\varphi_{a}$ is a bijection on $\operatorname{supp}(a)$, we called $\varphi_{a}$ a support permutation of $a$.

Let $a=\sum_{i=1}^{n} g_{j_{i}} \in F_{2} G$. Note that $a^{2}=a$ if and only if $\left(\sum_{i=1}^{n} g_{j_{i}}\right)^{2}=\sum_{i=1}^{n} g_{j_{i}}$. Since $\operatorname{char}\left(F_{2}\right)=2,\left(\sum_{i=1}^{n} g_{j_{i}}\right)^{2}=\sum_{i=1}^{n} g_{j_{i}}^{2}+\sum_{i<k} g_{j_{i}} g_{j_{k}}+\sum_{i>k} g_{j_{i}} g_{j_{k}}=\sum_{i=1}^{n} g_{j_{i}}^{2}+\sum_{i<k} g_{j_{i}} g_{j_{k}}+$ $\sum_{i<k} g_{j_{i}} g_{j_{k}}=\sum_{i=1}^{n} g_{j_{i}}^{2}+2 \sum_{i<k} g_{j_{i}} g_{j_{k}}=\sum_{i=1}^{n} g_{j_{i}}^{2}$ Thus, we have $\left(\sum_{i=1}^{n} g_{j_{i}}\right)^{2}=\sum_{i=1}^{n} g_{j_{i}}$ if and only if $\sum_{i=1}^{n} g_{j_{i}}^{2}=\sum_{i=1}^{n} g_{j_{i}}$. Thus, $a^{2}=a$ if and only if $\sum_{i=1}^{n} g_{j_{i}}^{2}=\sum_{i=1}^{n} g_{j_{i}}$. Hence we have proved the following result:

Proposition 2.3. Let $a \in F_{2} G$. Then $a \in I(G)$ if and only if $\varphi_{a}$ is a support permutation of $a$. 

unique.

In addition, the support permutation $\varphi_{a}$ of each idempotent $e \in I(G)$ is

Corollary 2.4. Let $g \in G$. If $\operatorname{ord}(g)=2$, then $g$ does not contain in the support of any idempotent.

Proof. Let $e \in I(G)$. Suppose that $g \in \operatorname{supp}(e)$ and $\operatorname{ord}(g)=2$.

Case 1: $1 \in \operatorname{supp}(e)$. Then, $\varphi_{e}(g)=\varphi_{e}(1)=1$, contrary to $\varphi_{e}$ is the support permutation of $e$.

Case 2: $1 \notin \operatorname{supp}(e)$. But $1 \in \operatorname{Im}\left(\varphi_{e}\right)$ as $\varphi_{e}(g)=1$, contrary to $\varphi_{e}$ is the support permutation of $e$.

Thus, $g \notin \operatorname{supp}(e)$.

Suppose that $e \in I(G)$ has the support permutation $\varphi_{e}$ which is a cycle of length $n$ in $S_{|G|}$ where $n=|\operatorname{supp}(e)|$. Then it is clear that for any arbitrary $g \in \operatorname{supp}(e)$

$$
e=g+\varphi_{e}(g)+\varphi_{e}\left(\varphi_{e}(g)\right)+\cdots+\varphi_{e}^{n-1}(g)=\sum_{i=1}^{n} g^{2^{i}-1}
$$

This observation motivates the following definition:

Definition 2.5. Let $e \in I(G)$ such that its support permutation $\varphi_{e}$ is a cycle of length $n$ in $S_{|G|}$ where $n=|\operatorname{supp}(e)|$. Then any element $g \in \operatorname{supp}(e)$ is called a generator of $e$ and $e$ is called a generated idempotent, denote as $e=\langle g\rangle$.

Note that 0 and 1 are the two trivial generated idempotents in every $F_{2} G$. On the other hand, it is obvious by Definition 2.5 that a non-trivial idempotent in $F_{2} G$ is not a generated idempotent if its support contains the identity $1 \in G$. In addition, each generated idempotent $e \in F_{2} G$ has exactly $n$ many distinct generators if $\varphi_{e}$ is a cycle of length $n$. Below are two immediate results indicated by Definition 2.5:

Proposition 2.6. Let $x \in G$. Then $x \in \operatorname{supp}(e)$ where $e=\langle g\rangle$ if and only if $x=g^{2^{j}}$ for some unique $j \in\{0,1,2, \ldots,|\operatorname{supp}(e)|-1\}$.

Proposition 2.7. Let $x \in G$. Then $x \in \operatorname{supp}(e)$ where $e=\langle g\rangle$ if and only if $g=x^{2^{j}}$ for some unique $j \in\{0,1,2, \ldots,|\operatorname{supp}(e)|-1\}$.

Also, it is clear that both Definition 2.5 and Proposition 2.6 implies the following result otherwise it will contradict Proposition 2.3

Proposition 2.8. Let $x \in G$. If $x \in \operatorname{supp}(e)$ where $e=\langle g\rangle$, then $\operatorname{ord}(x)=\operatorname{ord}(g)$.

Since a cycle can never be written as a product of shorter disjoint cycles, it is obvious that the support of any generated idempotent will not contain any other idempotent's. More detailed, we show that the supports of two distinct generated idempotents are mutually disjoint. 
Proposition 2.9. Let $e_{1}=\left\langle g_{1}\right\rangle$ and $e_{2}=\left\langle g_{2}\right\rangle$ be two distinct generated idempotents in $F_{2} G$, then $\operatorname{supp}\left(e_{1}\right) \cap \operatorname{supp}\left(e_{2}\right)=\emptyset$.

Proof. Suppose that $\operatorname{supp}\left(e_{1}\right) \cap \operatorname{supp}\left(e_{2}\right) \neq \emptyset$. Then by Proposition 2.6, for every $x \in \operatorname{supp}\left(e_{1}\right) \cap \operatorname{supp}\left(e_{2}\right), x=g_{1}^{2_{1}}$ and $x=g_{2}^{2^{j_{2}}}$ for some unique $j_{1} \in$ $\left\{0,1,2, \ldots,\left|\operatorname{supp}\left(e_{1}\right)\right|-1\right\}$ and $j_{2} \in\left\{0,1,2, \ldots,\left|\operatorname{supp}\left(e_{2}\right)\right|-1\right\}$. On the other hand, both Proposition 2.8 and Definition 2.5 indicate that $\operatorname{ord}(x)=\operatorname{ord}\left(g_{1}\right)=$ $\operatorname{ord}\left(g_{2}\right)$ and $\langle x\rangle=\left\langle g_{1}\right\rangle=\left\langle g_{2}\right\rangle$, which then lead to $e_{1}=e_{2}$, a contradiction. Thus, $\operatorname{supp}\left(e_{1}\right) \cap \operatorname{supp}\left(e_{2}\right)=\emptyset$.

The notion of generated idempotent will completely formularize all non-zero idempotents in as illustrated in Theorem 2.10 below.

Theorem 2.10. Every non-zero idempotent in $F_{2} G$ is either a generated idempotent or a finite sum of generated idempotents.

Proof. Let $e$ be a non-zero idempotent in $F_{2} G$ with its support permutation $\varphi_{e}$. If $e=1$, then $e$ is a trivial generated idempotent. Let $e$ be a non-trivial idempotent. Then $|\operatorname{supp}(e)|>1$ by Corollary 2.4 .

Case 1: If $\varphi_{e}$ is a cycle with length equal to $|\operatorname{supp}(e)|$, then $e$ is a generated idempotent.

Case 2: Suppose that $\varphi_{e}$ is a product of disjoint cycles, says $\varphi_{e}=\varphi_{1} \varphi_{2} \cdots \varphi_{k}$ such that the sum of all the length of $\varphi_{i}$ for $i=1,2, \ldots, k$ is equal to $|\operatorname{supp}(e)|$. Let $e_{i} \in F_{2} G$ such that $\operatorname{supp}\left(e_{i}\right) \subset \operatorname{supp}(e)$ and having $\varphi_{i}$ as its support permutation. By Definition 2.5, each $e_{i}$ is a generated idempotent. By Proposition 2.9, $e=\sum_{i=1}^{k} e_{i}$ and thus it is a finite sum of the generated idempotents $e_{i}$ for $i=1,2, \ldots, k$.

Proposition 2.1 and Theorem 2.10 indicate that knowing all the generated idempotents in $F_{2} G$ guarantees the full identification of the elements in $I(G)$. However, identifying all the generated idempotents in $F_{2} G$ is not an easy task for $G$ with large cardinality. Since the support of all generated idempotents are mutually disjoint and $I(G)$ is closed under addition, there exists a largest finite sum of all generated idempotents in $F_{2} G$.

Definition 2.11. The finite sum of all generated idempotents in $F_{2} G$ is called the largest idempotent in $F_{2} G$, denoted as $e_{L_{G}}$.

Clearly, $e_{L_{G}}$ is unique for each $F_{2} G$. Hence, we have the following result:

Theorem 2.12. The largest idempotent exists and is unique in every $F_{2} G$.

In the next section, the support of $e_{L_{G}}$ in every $F_{2} G$ will be identified and partitioned into disjoint supports of all non-zero generated idempotents. 


\section{Identification of All Idempotents}

This section is devoted to our main results. We first prove that $e_{L_{G}}=1$ for every $F_{2} G$ with $|G|=2^{\alpha}, \alpha \in \mathbb{Z}^{+} \cup\{0\}$. Next, we identify every $e_{L_{G}}$ where $|G|$ has an odd factor greater than one. For these cases, we further introduce a method to partition the support of $e_{L_{G}}$ in order to fully obtain all the non-zero generated idempotents in $F_{2} G$ and thus all the idempotents in $F_{2} G$.

It is obvious that the generator of every generated idempotent in $F_{2} G$ will also be a generator of a cyclic subgroup of $G$. Next, we further proof that the cyclic subgroup generated by a generator of a non-trivial generated idempotent in $F_{2} G$ must be non-trivial and of odd order.

Theorem 3.1. For $g \in G,\langle g\rangle$ is a non-trivial generated idempotent if and only if $\operatorname{ord}(g)$ is odd and $\operatorname{ord}(g)>1$.

Proof. Suppose that $\operatorname{ord}(g)$ is odd and $\operatorname{ord}(g)>1$, then there exist a smallest $k \in \mathbb{Z}^{+}$and $k>1$ such that $g^{2^{k}}=g$. Thus, $\left\{g, g^{2}, g^{4}, \ldots, g^{2^{k-1}}\right\}$ forms a support of a non-trivial generated idempotent $\langle g\rangle$ by Proposition 2.6.

Conversely, suppose there exists a non-trivial generated idempotent $\langle g\rangle$ such that $\operatorname{ord}(g)=2 k$ for some $k \in \mathbb{Z}^{+}$. By Corollary 2.4, we have $k>1$. Also by Definition 2.5 , then there exists a smallest $j \in \mathbb{Z}^{+}$and $j>1$ such that $g=g^{2^{j}}$. Thus, we have $1 \equiv 2^{j}(\bmod 2 k)$. Solving the congruence equation, we yield $2^{j}-1=2 l k$, for some $l \in \mathbb{Z}^{+}$. This is a contradiction since the left hand side of the equation is always odd as $j>1$ and the right hand side of the equation is always even. Hence, $\operatorname{ord}(g)$ must be odd and greater than one.

Theorem 3.1 above further suggests that the support of every non-trivial generated idempotent is contained in a non-trivial odd order subgroup of $G$. Note that if $|G|=2^{\alpha}$ for some $\alpha \in \mathbb{Z}^{+}$, then $G$ will not have any non-trivial odd order subgroup. Thus, we have the following result:

Corollary 3.2. if $|G|=2^{\alpha}$ for some $\alpha \in \mathbb{Z}^{+}$, then $F_{2} G$ has only trivial idempotents.

Next, we continue to study the idempotents in where has an odd factor greater than one. Suppose that $|G|$ has exactly $r$ many distinct odd prime factors for some $r \in \mathbb{Z}^{+}$. Then, $|G|=2^{\alpha} p_{1}^{\beta_{1}} p_{2}^{\beta_{2}} \cdots p_{r}^{\beta_{r}}$ for some $\alpha \in \mathbb{Z}^{+} \cup\{0\}$ and some $\beta_{1}, \beta_{2}, \ldots, \beta_{r} \in \mathbb{Z}^{+}$. By the Fundamental Theorem of Finite Abelian Group, $G \simeq$ $H \otimes K$, where $|H|=2^{\alpha}$ and $|K|=p_{1}^{\beta_{1}} p_{2}^{\beta_{2}} \cdots p_{r}^{\beta_{r}}$. It is clear that this $K$ is the unique non-trivial subgroup of $G$ having the largest odd order. From now on, we denote this subgroup $K$ of $G$ having the largest odd order as $H_{L}$ and also if $H$ is a subgroup of $G$, we denote the group ring element $\sum_{g \in H} g$ as $\bar{H}$.

Theorem 3.3. If $|G|$ has an odd factor greater than one, then $e_{L_{G}}=\bar{H}_{L}$.

Proof. Since $\left|H_{L}\right|$ is odd, we have $\bar{H}_{L}^{2}=\bar{H}_{L} \bar{H}_{L}=\left(\sum_{g \in H_{L}} g\right) \bar{H}_{L}=\sum_{g \in H_{L}}\left(g \bar{H}_{L}\right)=$ $\left|H_{L}\right| \bar{H}_{L}=\bar{H}_{L}$. Thus $\bar{H}_{L}$ is an idempotent. Next we show that $\bar{H}_{L}$ is a finite sum 
of generated idempotents. Note that $1 \in \operatorname{supp}\left(\bar{H}_{L}\right)=H_{L}$ and since $1^{2^{i}}=1$ for any $i \in \mathbb{Z}^{+}$, we know that $\varphi_{\bar{H}_{L}}$ will not be a cycle and thus by Theorem $2.10, \bar{H}_{L}$ is a finite sum of at least two generated idempotents. Lastly, we claim that the support of every generated idempotent contains in $H_{L}$. Suppose that there exists $g \in G-\{1\}$ such that $\langle g\rangle$ is a non-trivial generated idempotent with $g \notin H_{L}$. Let $H$ be the cyclic subgroup of $G$ generated by $g$. Then by Theorem 3.1, $H$ must be odd. Note that $H \cap H_{L}$ is a proper subgroup of $H$ as $g \notin H_{L}$. Hence, $H H_{L}$ is another subgroup of $G$ having odd order $\left|H H_{L}\right|$ that is greater than $\left|H_{L}\right|$ as $\left|H H_{L}\right|=\frac{|H|\left|H_{L}\right|}{\left|H \cap H_{L}\right|}$ and $\frac{|H|}{\left|H \cap H_{L}\right|}>1$. This is a contrary to $H_{L}$ is the largest odd order subgroup of $G$. Thus, every generated idempotent's support is contained in $H_{L}$. By Proposition 2.9 and Theorem 2.10, we have shown that $e_{L_{G}}=\bar{H}_{L}$.

By the Fundamental Theorem of Finite Abelian Group, $H_{L} \simeq \bigotimes_{i=1}^{k} C_{i}$ for some $k \in \mathbb{Z}^{+}$, where $C_{i}=\left\langle a_{i} \mid a_{i}^{n_{i}}=1\right\rangle$ and $n_{i}$ is odd for every $i \in\{1,2, \ldots, k\}$. Then, every group element $g \in H_{L}$ can be written in the form of $g=\prod_{i=1}^{k} a_{i}^{m_{i}}$ where $0 \leq m_{i}<n_{i}$ for every $i \in\{1,2, \ldots, k\}$. Recall the group isomorphism $\phi: H_{L} \rightarrow \bigotimes_{i=1}^{k} \mathbb{Z}_{n_{i}}$ such that $\phi\left(\prod_{i=1}^{k} a_{i}^{m_{i}}\right)=\left(m_{1}, m_{2}, \ldots, m_{k}\right)$. In order to partition $H_{L}$ to obtain the support of all the disjoint generated idempotents, it is the same as partitioning $\operatorname{Im}(\phi)$ into the isomorphic form of the generated idempotents' support.

Let $\left(m_{1}, m_{2}, \ldots, m_{k}\right),\left(r_{1}, r_{2}, \ldots, r_{k}\right) \in \operatorname{Im}(\phi)$, define a relation such that $\left(m_{1}, m_{2}, \ldots, m_{k}\right) \sim\left(r_{1}, r_{2}, \ldots, r_{k}\right)$ if and only if there exists $j \in \mathbb{Z}^{+} \cup\{0\}$ such that for every $i \in\{1,2, \ldots, k\}, m_{i}=2^{j} r_{i}\left(\bmod n_{i}\right)$. It can be checked easily that $\sim$ is an equivalence relation on $\operatorname{Im}(\phi)$. Thus, the set $\operatorname{Im}(\phi)$ is partitioned by $\sim$ and each of the partition is named by using definition below.

Definition 3.4. The set $K_{\left(m_{1}, m_{2}, \ldots, m_{k}\right)}=\left\{\left(r_{1}, r_{2}, \ldots, r_{k}\right) \in \operatorname{Im}(\phi) \mid\left(r_{1}, r_{2}, \ldots, r_{k}\right) \sim\right.$ $\left.\left(m_{1}, m_{2}, \ldots, m_{k}\right)\right\}$ is called the 2-generalized cyclotomic coset of $\operatorname{Im}(\phi)$ containing $\left(m_{1}, m_{2}, \ldots, m_{k}\right)$.

Next, we show that each 2-generalized cyclotomic coset of $\operatorname{Im}(\phi)$ induces a generated idempotent in $F_{2} G$.

Theorem 3.5. Suppose that $K_{1}, K_{2}, \ldots, K_{l}$ are all the 2-generalized cyclotomic cosets of $\operatorname{Im}(\phi)$, then there are exactly $l$ many generated idempotents in $F_{2} G$, namely $e_{1}, e_{2}, \ldots, e_{l}$ such that each $e_{i}=\sum_{\left(m_{1}, m_{2}, \ldots, m_{k}\right) \in K_{i}} \prod_{j=1}^{k} a_{j}^{m_{j}}$.

Proof. Let $K_{\left(m_{1}, m_{2}, \ldots, m_{k}\right)}$ be a 2-generalized cyclotomic coset. If $\left(r_{1}, r_{2}, \ldots, r_{k}\right) \in$ $K_{\left(m_{1}, m_{2}, \ldots, m_{k}\right)}$, then there exists $j \in \mathbb{Z}^{+} \cup\{0\}$ such that for every $i \in\{1,2, \ldots, k\}$, $m_{i}=2^{j} r_{i}\left(\bmod n_{i}\right)$. That is, $\prod_{i=1}^{k} a_{i}^{m_{i}}=\prod_{i=1}^{k} a_{i}^{2^{j}} r_{i}=\left(\prod_{i=1}^{k} a_{i}^{r_{i}}\right)^{2^{j}}$. Then by Proposition 2.7, we have $\prod_{i=1}^{k} a_{i}^{r_{i}} \in \operatorname{supp}\left(\left\langle\prod_{i=1}^{k} a_{i}^{m_{i}}\right\rangle\right)$ if and only if $\phi\left(\prod_{i=1}^{k} a_{i}^{r_{i}}\right) \in K_{\left(m_{1}, m_{2}, \ldots, m_{k}\right)}$. 
Hence, $\phi^{-1}\left(K_{\left(m_{1}, m_{2}, \ldots, m_{k}\right)}\right)=\operatorname{supp}\left(\left\langle\prod_{i=1}^{k} a_{i}^{m_{i}}\right\rangle\right)$. Thus, each of the group ring element $e_{i}=\sum_{\left(m_{1}, m_{2}, \ldots, m_{k}\right) \in K_{i}} \prod_{j=1}^{k} a_{j}^{m_{j}}$ is a generated idempotent for every $i \in$ $\{1,2, \ldots, k\}$.

On the other hand, let $e=\left\langle\prod_{i=1}^{k} a_{i}^{m_{i}}\right\rangle$. Then by Definition 2.5 and Proposition 2.7 , for every $x=\prod_{i=1}^{k} a_{i}^{r_{i}} \in \operatorname{supp}(e)$, there exists a unique $j \in\{0,1,2, \ldots, k-1\}$ such that $\prod_{i=1}^{k} a_{i}^{m_{i}}=x^{2^{j}}=\left(\prod_{i=1}^{k} a_{i}^{r_{i}}\right)^{2^{j}}=\prod_{i=1}^{k} a_{i}^{2^{j}} r_{i}$. This is equivalent to for every $x=\prod_{i=1}^{k} a_{i}^{r_{i}} \in \operatorname{supp}(e)$, there exists a unique $j \in\{0,1,2, \ldots, k-1\}$ such that for every $i \in\{1,2, \ldots, k\}, m_{i}=2^{j} r_{i}\left(\bmod n_{i}\right)$. Thus, $\operatorname{supp}(e)=\left\{\prod_{i=1}^{k} a_{i}^{r_{i}} \mid\left(r_{1}, r_{2}, \ldots, r_{k}\right) \in\right.$ $\left.K_{\left(m_{1}, m_{2}, \ldots, m_{k}\right)}\right\}$.

Note that result below further assure that the generated idempotents are generalization from our basis idempotents in [7].

Corollary 3.6. Let $\left\{e_{1}, e_{2}, \ldots, e_{l}\right\}$ be the set of all generated idempotents in $F_{2} G$. Then, $\left\{b_{1} e_{1}+b_{2} e_{2}+\cdots+b_{l} e_{l} \mid b_{i} \in F_{2}\right\}$ is the set of all idempotents in $F_{2} G$. Moreover, we have $|I(G)|=2^{l}$.

The following examples illustrate the full identification of idempotents in some $F_{2} G$ :

Example 3.7. Consider the binary group ring $F_{2} G$ where $G=C_{1} \otimes C_{2}$ such that $C_{1}=\left\langle a_{1} \mid a_{1}^{3}=1\right\rangle$ and $C_{2}=\left\langle a_{2} \mid a_{2}^{9}=1\right\rangle$. Clearly, $G$ itself is exactly $H_{L}$. By Theorem 3.3, $G$ forms the support of the largest idempotent $e_{L_{G}}$. Using Definition 3.4, we yield all the 2-generalized cyclotomic cosets of $\phi(G)$.

$K_{(0,0)}=\{(0,0)\}, K_{(1,0)}=\{(1,0),(2,0)\}$,

$K_{(0,1)}=\{(0,1),(0,2),(0,4),(0,5),(0,7),(0,8)\}, K_{(1,3)}=\{(1,3),(2,6)\}$,

$K_{(0,3)}=\{(0,3),(0,6)\}, K_{(1,1)}=\{(1,1),(2,2),(1,4),(2,5),(1,7),(2,8)\}$,

$K_{(2,3)}=\{(2,3),(1,6)\}, K_{(1,2)}=\{(2,1),(1,2),(2,4),(1,5),(2,7),(1,8)\}$.

By Theorem 3.5, we yield the corresponding generated idempotents:

$e_{1}=1, e_{2}=a_{1}+a_{1}^{2}, e_{3}=a_{2}+a_{2}^{2}+a_{2}^{4}+a_{2}^{5}+a_{2}^{7}+a_{2}^{8}, e_{4}=a_{1} a_{2}^{3}+a_{1}^{2} a_{2}^{6}$, $e_{5}=a_{2}^{3}+a_{2}^{6}, e_{6}=a_{1} a_{2}+a_{1}^{2} a_{2}^{2}+a_{1} a_{2}^{4}+a_{1}^{2} a_{2}^{5}+a_{1} a_{2}^{7}+a_{1}^{2} a_{2}^{8}, e_{7}=a_{1}^{2} a_{2}^{3}+a_{1} a_{2}^{6}$, $e_{8}=a_{1}^{2} a_{2}+a_{1} a_{2}^{2}+a_{1}^{2} a_{2}^{4}+a_{1} a_{2}^{5}+a_{1}^{2} a_{2}^{7}+a_{1} a_{2}^{8}$. The set of all idempotents in $F_{2} G$ is $I(G)=\left\{\sum_{i=1}^{8} b_{i} e_{i} \mid b_{i} \in F_{2}\right\}$. 
Example 3.8. Consider the binary group ring $F_{2} G$ where $G=C_{1} \otimes C_{2} \otimes C_{3}$ such that $C_{1}=\left\langle a_{1} \mid a_{1}^{2}=1\right\rangle, C_{2}=\left\langle a_{2} \mid a_{2}^{3}=1\right\rangle$ and $C_{3}=\left\langle a_{3} \mid a_{3}^{6}=1\right\rangle$. Clearly, $H_{L}$ is $C_{2} \otimes C_{3}^{\prime}$ which is of order 9 , where $C_{3}^{\prime}$ is the subgroup of $C_{3}$ generated by $a_{3}^{2}$. Thus, the largest idempotent $e_{L_{G}}$ has support $C_{2} \otimes C_{3}^{\prime}$. Using Definition 3.4 to partition $\phi\left(C_{2} \otimes C_{3}^{\prime}\right)$, we have the following 2-generalized cyclotomic cosets:

$K_{(0,0)}=\{(0,0)\}, K_{(1,0)}=\{(1,0),(2,0)\}, K_{(0,1)}=\{(0,1),(0,2)\}$,

$K_{(1,1)}=\{(1,1),(2,2)\}, K_{(1,2)}=\{(1,2),(2,1)\}$. Each of them induces a generated idempotent by Theorem 3.5, which are $e_{1}=1, e_{2}=a_{2}+a_{2}^{2}, e_{3}=a_{3}^{2}+a_{3}^{4}, e_{4}=$ $a_{2} a_{3}^{2}+a_{2}^{2} a_{3}^{4}$ and $e_{5}=a_{2} a_{3}^{4}+a_{2}^{2} a_{3}^{2}$ respectively. The set of all idempotents in $F_{2} G$, is exactly $I(G)=\left\{\sum_{i=1}^{5} b_{i} e_{i} \mid b_{i} \in F_{2}\right\}$.

\section{Concluding Remarks}

The intention to discover the potential of zero-divisor codes having idempotent generator triggered us to identify all the idempotents in binary abelian group rings. In this paper, we introduced the notion of generated idempotent and proved some important properties of these idempotents that enable the identification of all the idempotents in any arbitrary binary abelian group ring. However, the fact that every idempotent is a generated idempotent or finite sum of generated idempotents is generally not true for binary non-abelian group rings.

Acknowledgement. This work was supported by Universiti Sains Malaysia (USM) Research University (RU) Grant no.1001/PMATHS/811286.

\section{REFERENCES}

[1] Berman, S.D., On the Theory of Group Codes, Kibernetika, 3 (1967), 31-39.

[2] Fu,W. and Feng, T., "On Self-orthogonal Group Ring Codes", Designs, Codes and Cryptography, 50 (2009), 203-214.

[3] Hurley, B. and Hurley, T., "Systems of MDS Codes from Units and Idempotents", Discrete Mathematics, 335 (2014), 81-91.

[4] Hurley, P. and Hurley, T., "Codes from Zero-divisors and Units in Group Rings", Int. J. Information and Coding Theory, 1 (2009), 57-87.

[5] Jitman, S., Ling, S., Liu, H. W. and Xie, X., "Abelian Codes in Principal Ideal Group Algebras", IEEE Transactions on Information Theory, 59 (2013), 3046-3058.

[6] McLoughlin, I. and Hurley, T., "A Group Ring Construction of The Extended Binary Golay Code", IEEE Transactions in Information Theory, 54 (2008), 4381-4383.

[7] Ong, K.L., Ang, M.H., "Study of Idempotents in Cyclic Group Rings over F2", AIP Conference Proceedings, 1739, 020011 (2016), doi: 10.1063/1.4952491.

[8] Tan, Z. S., Ang, M. H. and Teh, W. C., "Group Ring Codes over a Dihedral Group", Accepted by Malaysian Journal of Mathematical Sciences, (2015).

[9] Wong, Denis C.K. and Ang, M.H., "Group Algebra Codes Defined over Extra Special pgroup", Journal of Algebra, Number Theory and Applications, 78 (2013), 19-27. 
[10] Wong, Denis C.K. and Ang, M.H., "A family of MDS abelian group Codes", Far East Journal of Mathematical Sciences, 78 (2013), 19-27.

[11] Wong, Denis C.K. and Ang, M.H., "Group Codes Define Over Dihedral Groups of Small Order", Malaysian Journal of Mathematical Sciences, 7(S) (2013), 101-116. 